\title{
Crecimiento económico: contribución y participación de los recursos
}

\author{
Economic growth: contribution y resources share \\ Daniel Villalobos Céspedes \\ Escuela de Administración, Universidad Nacional, Costa Rica \\ daniel.villalobos.cespedes@una.cr
}

Recibido: 08/10/2018 • Aceptado: 18/09/2019

\section{RESUMEN}

Esta investigación pretende ser un aporte a la teoría del crecimiento económico. Se plantea un análisis conceptual lógico-matemático que da origen a un modelo riguroso potencialmente útil. Se parte del costo de producción y se deriva la elasticidad de composición de los recursos, que incluye la del capital-trabajo a cada instante, las cuales sustentan los resultados fundamentales del modelo. Se devela la contribución y participación de los recursos al crecimiento del costo y de la producción. La ganancia y su tasa constituyen un aporte del trabajo y del capital global. Se devela un residuo que emana de la inversión y se expresa en la producción y la ganancia. Dentro de límites relevantes, se evalúa el modelo con base en los índices de capital fijo, trabajo y producción de la manufactura de Estados Unidos (1899-1922), aportados por Cobb y Douglas (1928) en A theory of production.

Palabras clave: recursos; composición; elasticidad; residuo.

\section{ABSTRACT}

This research seeks to contribute to the economic growth theory. It proposes a logical-mathematical conceptual analysis, 
generating a rigorous and useful model of economic growth. The starting point is the cost of production from which the elasticity of resources' composition, which includes the elasticity of capital-labor composition of the production process, is among the fundamental results. It was unveiled the contribution and share of resources in the cost and on production growth. From that dynamic, resources as the profit and the rate of profit appear as labor and capital contribution. It is revealed a residue which originates in investment and is reflected by the production and profit. To appraise the model, within relevant limits, we found useful capital, labor, and production indexes of the United States' Manufacture (1899-1922) provided by Cobb and Douglas (1928) in A Theory of Production.

Key Words: resources; composition; elasticity; residue.

\section{INTRODUCCIÓN}

Formalmente, la teoría del crecimiento económico tuvo su origen en $A$ theory of production de Cobb y Douglas (1928). Estos autores centran su investigación en la manufactura de Estados Unidos durante los años (1899-1922), para lo cual construyen indicadores de producción, capital y trabajo. Con ello, miden el crecimiento de la producción según variaciones en el nivel de capital y trabajo. Del análisis de relaciones entre esas variables derivan la contribución de esos recursos al crecimiento del producto. La función de producción de esos autores es homogénea de grado uno del capital y del trabajo y la producción tiende a cero cuando la cantidad de uno de los recursos tiende a cero. Los autores suponen constante la elasticidad del producto con respecto de pequeñas variaciones en el nivel de trabajo $(0<\alpha<1)^{1}$ y del capital $(1-\alpha)$, razón por la cual puede tomarse como un parámetro puramente tecnológico (Piketty, 2014, p. 218).

Cobb y Douglas subrayan que a sus resultados se les ha dado un valor numérico exacto para poder fijar la idea, pero los números mismos se fijan provisionalmente en relación con determinado periodo y a ciertos índices. Cuando los índices sean refinados o el período cambia, puede ser que la constante $3 / 4$ aparezca como una constante .7, .6 o tal vez como una

1 No obstante $\alpha=3 / 4$ no es una asignación antojadiza de esos autores, se basa en el índice estimado $(\alpha=0.74)$ por la Oficina Nacional de Investigación Económica para el período (1909-1918) para la manufactura de los Estado Unidos (Cobb \& Douglas, 1928). 
variable (Cobb \& Douglas, 1928). Así, para fijar la idea, $(\alpha)$ se supone constante e igual a $3 / 4$; esto significa que pequeñas variaciones porcentuales solamente en el trabajo, tienen tres veces el efecto que se produciría por el mismo cambio porcentual solamente en el capital" (Cobb \& Douglas, 1928 , p. 156). Además, indican esos autores que se debe (1) estar preparado para idear fórmulas que no necesariamente se basen en la "contribución" relativa constante de cada factor al producto total, sino que permitan variaciones anuales, y (2) eliminar hasta donde sea posible el elemento tiempo de los procesos (Cobb \& Douglas, 1928, p. 165). Solow (1957) y Arrow, Chenery, Minhas y Solow (1961) ofrecen un sistemático esfuerzo teórico en esta dirección, mientras Villalobos (2019) sugiere que $(\alpha)$ varía a cada instante. Si bien el modelo de contabilidad del crecimiento permite variaciones de $(\alpha)$ en el rango $(0<\alpha<1)$ (Barro \& Sala-i-Martin, 2004; Crafts, 2009; Abramovitz, 1956; 1962; Hulten, 2009)², el procedimiento no corresponde con el método y las sugerencias de Cobb y Douglas (1928).

La primera sección de esta investigación se plantea un modelo de crecimiento económico según participación de los recursos en el crecimiento de los costos (Villalobos, 2019). Del segundo al cuarto apartado, el objetivo es medir el consumo o depreciación, remanente, reposición y acumulación por nueva inversión del capital fijo, el capital laboral y el trabajo, en términos de producto. La siguiente sección se plantea la posibilidad de un residuo con origen en los costos de producción. Las funciones de producción y ganancia trazadas en las secciones sexta y sétima, siguen el método con el cual se deriva la función de costos y se formula la contribución y participación de los recursos en el producto. La cuantificación de la depreciación y el remanente se desarrolla en el octavo apartado. La penúltima sección presenta las conclusiones, implicaciones y posibles líneas de investigación. En el anexo se intenta evaluar la rigurosidad y fortaleza de los supuestos críticos de la propuesta teórica ${ }^{3}$, al utilizar los índices de producción, capital y trabajo generados por Cobb y Douglas (1928).

2 Abramovitz (1956) se propuso analizar las tendencias de los recursos y el producto en la economía de los Estados Unidos de América desde $1870 \mathrm{y}$, a tal efecto, se formuló tres preguntas que contienen, en general, las preocupaciones de Cobb y Douglas (1928); empero el método analítico difiere al de estos últimos autores.

3 Solow (1956) sugirió que toda teoría depende de supuestos que no son completamente ciertos, pero las conclusiones dependen sensiblemente de supuestos cruciales, razonablemente realísticos. Así, si los supuestos son dudosos, los resultados son sospechosos (p. 65). 
Esta investigación formula la cuantificación de los valores $\forall\{\alpha, \alpha, \alpha, \alpha, \alpha:(0,1)\}$ a cada instante, e.g. anual, y plantea la equivalencia costo-ingreso medio del trabajo como resultado de las variaciones en la elasticidad de composición de los recursos. La posibilidad de medir la viabilidad tecnológica al introducir el capital de trabajo, como materias primas o insumos, como variables del modelo de costos, permite concluir que esta no necesariamente desplaza trabajo. Se propone una medida de la elasticidad de depreciación y de la composición de los recursos en general y del capital-trabajo en particular. La diferencia entre costo relativo potencial y efectivo, podría revelar un residuo como efecto tecnológico y de las condiciones de la economía.

Dentro de los límites que fija la serie de índices provistos por Cobb y Douglas (1928), un resultado práctico de esta investigación, relegado al anexo, es la medición de la contribución y participación de los recursos en el crecimiento de los costos, el producto y la ganancia. Se supone que esos índices se expresan en valor real, así el precio del capital y el salario están implícitos. Según Cobb-Douglas (1928), "el propósito de este escrito (...) no es establecer resultados, sino ilustrar un método de ataque" (p. 156) al problema de la contribución y participación de los recursos en el crecimiento económico.

\section{La función de costo de producción}

Una economía afronta costos de producción $(C)$ a cada instante, por ejemplo, anualmente. En general, esos costos pueden clasificarse según tipos de recursos que sustentan la inversión; fijo K, variables Ḱ -materias primas e insumos- y trabajo $L$. La función de costos en una economía puede ser:

(1) $\mathrm{C}=\mathrm{F}(\mathrm{K}, \mathrm{K}, L)$

Si $\mathrm{K}=\frac{\mathrm{K}}{\mathrm{K}}$ mide la viabilidad tecnológica o capacidad de la economía para transformar $(\mathrm{K}, \mathrm{K})$ al transferir sus atributos de valor al producto $(Y)$, la ecuación (1) se redefine:

(2) $C=F[(1, \mathrm{\kappa}) \mathrm{K} ; L]$

Sea $1=F\left[(1, \kappa) \frac{K}{C}, \frac{L}{C}\right]$ y sea $(\kappa=0)$ para que $1=F\left(\frac{K}{C}, \dot{\alpha}\right) \therefore 1-F(\hat{\alpha})=F\left(\frac{K}{C}\right)$ tal que $\alpha=\frac{L}{c} ; \forall\{\alpha \dot{\alpha}:(0,1)\}$ es la contribución media del trabajo en el costo y así $[1=F[\alpha,(1-\alpha)]]$. 
Al diferenciar la ecuación (2) y al simplificar:

(3) $\dot{c}=\frac{f[[\hat{\mathrm{k} \kappa},(1, \mathrm{\kappa}) k] \mathrm{K} ; n L]}{F[(1, \mathrm{\kappa}) \mathrm{K} ; L]}$

y $(c, n, k, \overline{\mathrm{k}})$ son las respectivas tasas de crecimiento relativo de $(C, L, \mathrm{~K}, \mathrm{~K})$. Sea $d \mathrm{~K}=d\left(\frac{K}{\mathrm{~K}}\right) \therefore$

(4) $\mathrm{k}=\mathrm{k}-k$

y

(5) $k=\mathrm{k}-\mathrm{k}$

Sin progreso tecnológico ( $(\mathrm{k}=0 ; k=\mathrm{k})$; dada la viabilidad tecnológica, la inversión en $\mathrm{K}, \hat{K}$ es proporcional. Si $\frac{\partial \mathrm{K}}{\partial \hat{K}}=\frac{1}{K} \therefore \hat{K}=\mathrm{k}$ tal que $\mathrm{K}$ se encuentra ociosa, su uso no es óptimo o bien existe exceso o escasez de inversión. Si $\frac{\partial \mathrm{K}}{\partial \mathrm{K}}=-k \mathrm{~K} \therefore \hat{\mathrm{K}}=-k$ se registra exceso o escasez de inversión en $\mathrm{K}, \mathrm{K}$; el exceso de un recurso podría evidenciar escasez en otros.

Al substituir las definiciones anteriores en la ecuación (3) y al simplificar:

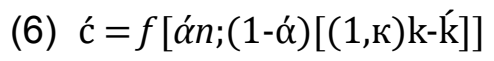

Se denota así la participación relativa de los recursos según influjo de la viabilidad tecnológica en la composición de los costos. Se precisa la participación proporcional de Ḱ,K dado (1-ó) y según incidencia de la viabilidad tecnológica, revelada por k,k; $d C=f\left[\alpha^{\prime} ;(1-\alpha)(1, \mathrm{k}) \mathrm{k},(\dot{\alpha}-1) \hat{\mathrm{k}}\right] C$ refleja cambio en el nivel de los costos a cada instante.

\section{Acumulación de capital fijo}

“(..) el reemplazo se queda corto de la depreciación (...), la inversión neta de depreciación no puede ser identificada con la inversión neta de reemplazo" (Domar, 1953, pp. 1-2). Suele suceder que K no se consume totalmente en cada instante, sino solo una fracción variable del mismo. Sea $\delta=\frac{\breve{\mathrm{K}}}{\mathrm{K}} \therefore \check{\mathrm{K}}=\delta \mathrm{K} ; \forall\{\delta:(0,1)\}$ el consumo productivo o depreciación $\breve{\mathrm{K}}$ de $\mathrm{K}$ transferida al producto $(Y)$. Sea $c=\frac{c}{Y} \therefore C=c Y$, donde $(c)$ es el costo medio de producción a cada instante. Ceteris paribus, $(C)$ varía conforme a $(Y)$ y 
una fracción қ de capital fijo aparece como acumulación efectiva, incluido el remanente: $\mathrm{қ}=\mathrm{K}, \mathrm{K}: . \mathrm{K}=\mathrm{K}-\delta \mathrm{K} .:$

(7) $\mathrm{қ}=(1-\delta) \mathrm{K}$

En la economía, las expectativas de ingresos podrían inducir inversiones en $\mathrm{K}$ que reflejen exceso de reposición, expansión y/o entradas y salidas ${ }^{4}$. La distribución del capital fijo según esa dinámica, se establece a partir de la ecuación (7): $\delta=\frac{\breve{\mathrm{K}}}{\mathrm{K}} ;(1-\delta)=\frac{\mathrm{K}}{\mathrm{K}} \therefore 1=\delta+(1-\delta)$. La función del capital fijo podría ser:

(8) $\mathrm{K}=F(\mathrm{~K}, \check{\mathrm{K}})$

Al diferenciar la ecuación previa, resulta $d \mathrm{~K}=d[F(\mathrm{~K}, \check{\mathrm{K}})] \therefore k=f\left(\mathrm{k} \frac{\mathrm{K}}{\mathrm{K}}, \mathrm{k} \frac{\mathrm{K}}{\mathrm{K}}\right)$, donde $\mathrm{k}, \mathrm{k}$ representan, respectivamente, la tasa de variación relativa del capital fijo remanente y de la depreciación efectiva. La tasa de variación relativa de la acumulación efectiva del capital fijo es:

(9) $k=f[\delta \mathrm{k},(1-\delta) \mathrm{k}]$

La acumulación relativa de capital fijo en cada período de producción se conjuga por la dinámica de la depreciación y el capital remanente debida a la reposición, ampliación, salida y entrada de capital. Si $k=0$, K, permanece igual y $-\delta \mathrm{k}=(1-\delta) \mathrm{k} \therefore \frac{\mathrm{k}}{\mathrm{k}}=\left|\frac{\delta}{1-\delta}\right|$ y sea $v=\frac{\mathrm{k}}{\mathrm{k}}$ para que:

(10) $v=\frac{\delta}{1-\delta}$

lo cual define la elasticidad de la depreciación y permite medir $\delta$ :

(11) $\delta=\frac{v}{1+v}$

Dado $\mathrm{k}=v \mathrm{k}, \quad \mathrm{y}$ al reemplazar en la ecuación (9) resulta $d \mathrm{~K}=d[F(\mathrm{~K}, \check{\mathrm{K}})] \therefore k=f\left(\mathrm{k}_{\mathbb{K}}^{\mathrm{K}}, \frac{\mathrm{k}}{\mathrm{K}}\right)$ y al insertar la definición (10):

(12) $k=2 \delta \mathrm{k}$

4 Al respecto, Keynes (1964) sugirió el criterio de demanda efectiva, sustantivo en los supuestos críticos de su teoría general del empleo. 
Se determina así la tasa de crecimiento de la acumulación efectiva del capital fijo equivalente solo a la reposición por depreciación a cada instante.

Al empotrar la ecuación (9) en la (5), la variación relativa del costo potencial es:

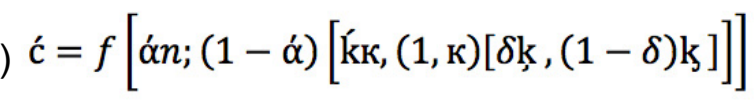

y por la ecuación (12):

$$
\text { (14) ć }=f\left[\alpha^{\prime} n ;(1-\alpha ́ \alpha)[\hat{\mathrm{k} \kappa},(1, \mathrm{\kappa}) 2 \delta \mathrm{k}]\right]
$$

Esta ecuación explica el rol del cambio relativo en la viabilidad tecnológica k y de la acumulación relativa en capital fijo $k$ como efecto neto de la variabilidad de la depreciación, la reposición, la ampliación y la entrada y salida de capital, en la composición de los recursos. La mayor productividad de los recursos se denotaría por la relación inversa entre el crecimiento relativo de la producción y de los costos medios, $C=F(c Y) \therefore \dot{c}=f(\dot{c}, \lambda)$ donde $\dot{c}, \lambda$ representan, en ese orden, la variación relativa de $(c, Y)$.

Al suponer $\dot{\mathrm{c}}=0$ en la ecuación (13) y al operar: $-f(\dot{\alpha} n)=f[(1-\dot{\alpha})[\mathrm{k} \kappa,(1, \kappa)[\delta \mathrm{k},(1-\delta) \mathrm{k}]]]:$.

(15) $\left|-\frac{\dot{\alpha}}{1-\dot{\alpha}}\right|=\frac{[\hat{\mathrm{k} \kappa},(1, \mathrm{\kappa})[\delta \mathrm{k},(1-\delta) \mathrm{k}]]}{n}$

Sea $v=\frac{\left[\mathrm{k \kappa}_{,}(1, \mathrm{k})\left[\delta \mathrm{k}_{,},(1-\delta) \mathrm{k}\right]\right]}{n}$ la elasticidad de composición del costo potencial, tal que:

(16) $v=\frac{\dot{\alpha}}{1-\dot{\alpha}}$

Y:

(17) $\alpha=\frac{v}{1+v}$ 
Sea $v n=[\hat{k} \kappa,(1, \kappa)[\delta \mathrm{k},(1-\delta) \mathrm{k}]]$ y al reemplazar en la ecuación (13) ocurre ć $=f[\alpha ́ n,(1-\alpha) v n]: \therefore c=f[\alpha ́,(1-\alpha ́) v] n$, y al substituir la ecuación (16) sucede $c=f(\alpha, \alpha) n:$

(18) $c=2 \alpha ́ n$

que define la tasa de crecimiento del costo efectivo de producción.

Por la ecuación (9) y (15) $v=\frac{[\mathrm{k \kappa},(1, \mathrm{k})[\delta \mathrm{k},(1-\delta) \mathrm{k}]]}{n} \therefore v=\left[\mathrm{k} \frac{\mathrm{K}}{n},(1, \mathrm{\kappa}) \frac{k}{n}\right]$ y sea $\mu=\frac{k}{n}$ la elasticidad de composición capital-trabajo, para que:

$$
\text { (19) } v=\left[\frac{\mathrm{k}}{n} \mathrm{\kappa} ; \mu(1, \mathrm{\kappa})\right]
$$

Si $\kappa=0$, lo cual simplifica la composición de los recursos en términos de $(\mathrm{K}, L)$, y dado $k=\mu n$, de la ecuación (13) o (14):

$$
\text { (20) ć }=f[\alpha ́ n,(1-\alpha) \mu n]
$$

Si $c=0$, entonces $-\alpha=(1-\alpha) \mu$.

(21) $(\mu, v)=\left|-\frac{\dot{\alpha}}{1-\dot{\alpha}}\right|$

Es evidente que $v$ revela la participación $\alpha, \delta$ de $K, \hat{K}, L$ y el influjo de $\mu$ en $C$. Estos resultados sugieren que en ć la asignación de los recursos se define según $v$, que incluye la participación del capital y el trabajo en el producto según $\mu$.

\section{Composición del costo de producción}

De la ecuación (2), la composición de los recursos $(r)$ en el costo de producción es:

$$
\text { (22) } r=(1, \mathrm{~K}) \frac{\mathrm{K}}{\mathrm{L}}
$$

En el largo plazo ( $r$ ) podría modificarse ceteris paribus a causa de cambios tecnológicos en $(K, K, K, L)$ y de la acumulación y depreciación de K, en cada instante. Sin embargo, en el corto plazo puede presentarse variabilidad en $(r)$ ceteris paribus según dinámica de los mercados y políticas de 
gobierno con influjo la velocidad de depreciación tal que $(k=0)$ y no habrá reposición, ni entradas ni salidas de capital. Al suponer $\kappa=0$ en la ecuación anterior, se define la composición del costo solo en términos de inversión en $(K, L)$ :

$$
\text { (23) } \hat{\mathrm{r}}=\frac{\mathrm{K}}{L}
$$

Al diferenciar $(\grave{\mathrm{r}}=k-n)$, donde $\ddot{\mathrm{r}}$ destaca la tasa relativa del cambio en $\hat{\mathrm{r}}$, tal que $(k=\ddot{r}+n)$ y la tasa de crecimiento relativo del costo registra la variabilidad neta de $\hat{\mathrm{r}}$ a cada instante. Dado $(k=\mu n)$, entonces $[\grave{\mathrm{r}}=\mu n-n \cdot: \grave{\mathrm{r}}=(\mu-1)$ n], y $\mu$ depende $(k, n)$ :

(24) $\mu=\frac{\ddot{\mathrm{r}}+n}{n}$

Al reemplazar este resultado en la ecuación (19):

(25) $v=\left[\frac{\hat{\mathrm{k}}}{n} \mathrm{~K} ;(1, \mathrm{~K})\left(\frac{\ddot{\mathrm{r}}+n}{n}\right)\right]$

Así, r̂ puede variar dentro de cierto límite en dirección opuesta a (к) en función de ḱ. Estos resultados se muestran explícitos al derivar la ecuación (22): '́r $r=\hat{\mathrm{k}} \kappa \frac{\mathrm{K}}{\mathrm{L}} ;(1, \mathrm{\kappa})(k-n) \frac{\mathrm{K}}{L} \therefore \dot{\mathrm{r}}=[\hat{\mathrm{k}} \kappa ;(1, \mathrm{\kappa}) \ddot{\mathrm{r}}] \frac{\hat{\mathrm{f}}}{r}$ y al insertar las ecuaciones (22) y (23):

(26) $\dot{r}=[\hat{k} \kappa ;(1, \kappa) \ddot{r}] \frac{1}{(1, \kappa)}$

Si $\hat{k}=0$, y por consiguiente la acumulación de $\mathrm{K}$, responde a la reposición por consumo o acumulación por reposición; [ŕr $\stackrel{\mathrm{r}}{=}=(\mu-1) n]$. Si $[\hat{\mathrm{k}} \neq 0 ; k=n: \mu=1 ; \grave{\mathrm{r}}=0]$, de la ecuación (26) se desprende:

(27) $\dot{r}=\frac{\kappa}{(1, \kappa)} \hat{k}$

Ello implica que la viabilidad tecnológica no necesariamente desplaza trabajo: si $[\grave{r}=0 ; k=n, \mu=1]$ y por la ecuación $(25) v>1: v=\left[\left(\frac{\mathrm{k}}{n}, 1\right) \kappa ; 1\right]$, y si además $\mathrm{k}=n: \therefore v=[(1,1) \kappa ; 1]: \therefore[v=(2 \kappa, 1)]>1$, y $(\mathrm{k})$ se duplica en razón de cada punto porcentual que varíe $(k, n)$, y cuando $(0<n<k), \stackrel{\mathrm{r}}{>}>0$ y $(\mu, v)>1$. Se revela en estos casos, con diferente grado de intensidad, la menor 
participación relativa de $(L)$ en la composición del costo. Empero, ello podría impulsar la productividad y la participación de los recursos en el crecimiento económico. Si $(0<k<n) ; \ddot{r}<0, \mu<1 ; v>0$ el nivel de trabajo crece. Si $(k, r)=0$, de la ecuación (26) emana $r$ $=0$; la composición del costo está estancada. No obstante, la ecuación (27) indica posibilidad de una participación equitativa del trabajo y el capital en el crecimiento económico dado $\hat{\mathrm{k}}>0 ; \mu=1$.

Si en la ecuación $(25) \hat{k}=0$ :

$$
\text { (28) } v=\left(\frac{\ddot{\mathrm{r}}+n}{n}\right)(1, \mathrm{\kappa}) \therefore v=\mu(1, \mathrm{\kappa})
$$

Así, $v$ podría oscilar dado $(1, \kappa)$ al fluctuar $\mu$. En tal caso, la participación del trabajo y el capital en el crecimiento económico podría ser desigual; favorece al capital si $\mu>1$. Además, si $(\grave{r}=0 ; \mu=1)$, de la ecuación anterior:

$$
\text { (29) } v=(1, \kappa)
$$

de donde, dada la viabilidad tecnológica y $(k=n)$, podría no variar $v \mathrm{y}$, sin embargo, $v>1 ; \mu=1$. Si, evento extremo, $(k<0, n=0)$, se indica una tasa de consumo de $\mathrm{K}$, en cada instante $(t)$ sin reposición y el nivel de $(L)$ es fijo; se registra una caída tendencial de ŕ proporcional a $(k<0)$, ḱ se deprime hasta un nivel indeterminado y $(v, \mu) \rightarrow 0)$. Además, ć, $(\rightarrow 0)$ si $(k<0, n=0)$ y $\dot{c}=f[(1-\alpha) k<0]$, a la que vez $\alpha(\rightarrow 1)$.

\section{Costo de producción potencial y efectivo}

Las mediciones de la variabilidad del costo reflejan el valor tendencial de $\alpha$ a cada instante, según tendencia de $(k, n, r$ r, r̈), con lo cual $(\mu, v)$ registran grados de respuesta ante variaciones en los recursos y la viabilidad tecnológica k. Sin embargo, $\alpha$ tiene que anclarse al valor de $(r)$ en cada instante. Esto es, $r_{t}=\left(1+\hat{r}_{t}\right) r_{t-1}$ es el momento del anclaje de $\alpha$ que permite el costo medio del trabajo en cada instante de la producción y su elasticidad respecto de las variaciones en el costo medio del capital. Al operar en la ecuación (22) e insertar el resultado en la ecuación (2), ocurre $C=F\left[(1, \mathrm{\kappa}) \frac{1}{(1, \mathrm{\kappa})} r L ; L\right] . \therefore$

$$
\text { (30) } C=F(r, 1) L
$$

Se deduce de esta ecuación que por cada unidad adicional de $(L)$, existe determinada $(r)$ en cada instante. Al diferenciar y suponer $(\hat{r}=0)$ : 18 
(31) $c_{L}=f[(r, 1) n] \alpha ́$

Por la ecuación (22) también se sabe que $L=\frac{1}{r}(1, \kappa)$ Y̧ y por la ecuación (2):

(32) $C=F\left[\left(1, \frac{1}{r}\right)(1, \kappa) \mathrm{K}\right]$

Al diferenciar y suponer $(\hat{k}, \hat{r})=0$ :

(33) $\dot{c}_{\mathrm{K}}=f\left[\left(\frac{r, 1}{r}\right)(1, \mathrm{\kappa}) k\right](1-\alpha)$

$\mathrm{Y}$ al equiparar las ecuaciones (33) y (31) se estima $\alpha$ efectivo a cada instante:

(34) $\stackrel{\alpha}{\alpha}=\frac{\mu(1, \kappa)}{r+\mu(1, \kappa)}$

\section{El residuo en la composición del costo}

El residuo (Solow, 1957; Arrow, Chenery, Minhas, \& Solow, 1961) o más allá de la contribución del capital y del trabajo (Swan, 1956) de la inversión, se puede estimar por la contribución de $(K, L)$ en ć en cada instante (Solow, 1956) y un estado dado de la técnica (Keynes, 1964) o dado el estado de las artes (Swan, 1956). De la ecuación (23) se derivan dos eventos equivalentes: $\left[\left(\mathrm{K}=\hat{\mathrm{r}} L ; L=\frac{1}{\hat{\mathrm{r}}} \mathrm{K}\right]\right.$. Al introducir estas definiciones en la ecuación (2), por separado se computan los costos medios de $(K, L)$ dado к. Así, por $(\underset{K}{\mathrm{~K}}=\hat{\mathrm{r}} L), C=F[(1, \mathrm{~K}) \hat{\mathrm{r}} L ; L] \therefore C=F[(1, \mathrm{~K}) \hat{\mathrm{r}} ; 1] L$ y sea $\hat{\mathrm{c}}_{L}=\frac{C}{L}$ el costo medio del trabajo, para que:

$$
\text { (35) } \hat{\mathrm{c}}_{L}=F[(1, \mathrm{~K}) \hat{\mathrm{r}} ; 1]
$$

Por $L=\frac{1}{\hat{\mathrm{r}}} \mathrm{K}, C=F\left[(1, \mathrm{~K}) \mathrm{K} ; \frac{1}{\mathrm{r}} \mathrm{K}\right] \therefore C=F\left[(1, \mathrm{~K}) ; \frac{1}{\hat{\mathrm{r}}}\right] \mathrm{K}$ y sea $\check{\mathrm{c}}_{\mathrm{K}}=\frac{C}{\mathrm{~K}}$ el costo medio del capital fijo, tal que:

$$
\text { (36) } \check{\mathrm{C}}_{\mathrm{K}}=F\left[(1, \kappa) ; \frac{1}{\hat{\mathrm{r}}}\right]
$$

El cociente de las ecuaciones (35) y (36) brinda como resultado el valor $\hat{r}$ efectivo en términos de los costos medios de los recursos en cada instante: $\frac{\hat{c}}{\check{c}}=\frac{F(\hat{r}, 1)}{F(\hat{r}, 1) \frac{1}{\hat{\mathrm{r}}}} \therefore$ 
(37) $\hat{\mathrm{r}}=\frac{\hat{\mathrm{c}}}{\mathrm{c}}$

Al derivar, se obtiene la tasa de variación neta de $\hat{r}$ inducida por variaciones relativas en la participación de los recursos en los costos medios:

(38) $\dot{\mathrm{r}}=\dot{\mathrm{c}}_{\mathrm{K}}-\dot{\mathrm{c}}_{L}$

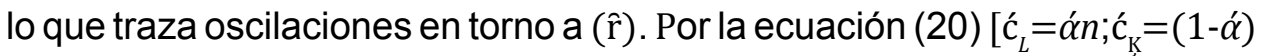
$k$ ] y por la (38) $\left(\dot{\mathrm{r}}, \dot{\mathrm{c}}_{L}\right)=\dot{\mathrm{c}}_{\mathrm{K}}$ tal que: ${ }^{5}$

(39) $c=2 \alpha ́ n+\dot{r}$

Sea r̀ el residuo, que resulta de la participación de los recursos en los costos efectivos y fluctúa acorde con $(\delta, n, \mathrm{k}, \mathrm{k})$ dado ḱ y por consiguiente $(v$, $v, \mu, \alpha, \alpha) ;$ Si $\dot{r}=0$ se obtiene la ecuación (18).

\section{La función de producción}

En criterio de Domar (1946), la productividad del trabajo no es una función del progreso tecnológico en abstracto, sino al progreso tecnológico incorporado en bienes de capital y a la cantidad de bienes de capital en general. Incluso sin progreso tecnológico, la acumulación de capital incrementa la productividad del trabajo, al menos hasta cierto punto; porque más capital es utilizado por trabajador en cada industria y debido a que hay un desplazamiento de trabajo hacia industrias intensivas en capital que pueden pagar salarios más altos. La producción es resultado de la transformación y transferencia de atributos de (K, Ḱ, $L$ ) por medio de procesos reflejan costos de producción. Empero, dado que se está intentando medir el capital que contribuye en la producción de bienes, se debe excluir el capital de trabajo, ya que es el resultado y no la causa del proceso de manufactura (Cobb \& Douglas, 1928).

Así, la función de producción sería:

5 Sea $d C=d L$ la condición equivalente del cambio en el costo total de producción $(C)$ debido a una unidad adicional de $(L)$, de modo que $\frac{d C}{d L}=1$. Así, $\frac{\dot{c}}{n} \frac{C}{L}=1 \therefore \frac{\dot{c}}{n}=\frac{L}{C}$ y dado que $\alpha^{\prime}=\frac{L}{C}$ entonces

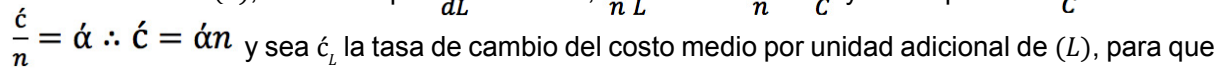
$\dot{c}_{L}=\alpha ́ n$. 
(40) $Y=F(K, L)$

Sea $1=F\left(\frac{K}{Y}, \frac{L}{Y}\right)$ y sea $\left[\alpha=\frac{L}{Y} \therefore(1-\alpha)=\frac{K}{Y}\right]$, y al diferenciar la ecuación (40) e insertar las definiciones anteriores, resulta la tasa de crecimiento producto potencial de la economía:

$$
\text { (41) } \lambda=[\alpha n ;(1-\alpha) k]
$$

No obstante, por la ecuación (23) se sabe que $\left[\mathrm{K}=\hat{\mathrm{r}} L ; L=\frac{1}{\hat{\mathrm{r}} \mathrm{K}}\right]$ y al substituir en la función (40) se desprenden dos funciones complementarias:

$$
\text { (42) } Y=F(\hat{r}, 1) L
$$

y por la derivada y dado $(\stackrel{\mathrm{r}}{=}=0)$ resulta la participación media del trabajo en el producto:

$$
\text { (43) } \lambda_{L}=f(\hat{\mathrm{r}}, 1) n \alpha
$$

Luego:

$$
\text { (44) } Y=F\left[\left(1, \frac{1}{\hat{\mathrm{r}}}\right) k \mathrm{~K}\right]
$$

Por la derivada y si $(\stackrel{\mathrm{r}}{=}=0)$ se deduce la participación media del capital en el producto:

$$
\text { (45) } \lambda_{\mathrm{K}}=f\left[\left(1, \frac{1}{\mathrm{r}}\right)(1-\alpha) k\right]
$$

Y al equiparar las ecuaciones (44) y (45):

$$
\text { (46) } \alpha=\frac{\mu}{\hat{\mathrm{r}}+\mu}
$$

y se computa $\alpha$ efectivo a cada instante de $(r)$ equivalente a la ecuación (34); si $(\alpha=\alpha)$ en un instante dado la participación del trabajo en la producción es idéntica a su participación en los costos. Respecto de la ecuación (34) implica que $(1, \kappa)=1$ y en ese caso se requiere el evento extremo $(\kappa=0)$. Además, si $[\mu=(\hat{\mathrm{r}}=r)]$ el ingreso del trabajo es igual al del capital.

\section{Participación de los recursos en el producto}


La contribución de los recursos en $(v)$ definida según [á,(1-ó)], podría diferir de su participación en los costos según su nivel en términos de valor. Sea $c=\frac{C}{Y}$ el costo medio de la producción tal que $C=c Y$, y al substituir en la ecuación (2) y al simplificar resulta:

$$
\text { (47) } c=F\left[(1, \kappa) \frac{\mathrm{K}}{Y} ; \frac{L}{Y}\right]
$$

Sea $\alpha=\frac{L}{Y}$ la contribución del trabajo en el nivel de producción, para que $(1-\alpha)=\frac{\mathrm{K}}{Y}$ ner $\kappa=0$ se obtiene:

$$
\text { (48) } \mathrm{c}=F[\alpha,(1-\alpha)]
$$

con lo cual se puede estimar el costo efectivo de la producción al reemplazar la definición de $c ; \frac{C}{Y}=F[\alpha,(1-\alpha)]$ de donde resulta la distribución de $(Y)$ entre los recursos en términos de costo-ingreso:

(49) $W=F[\alpha,(1-\alpha)] Y$

donde $W$ representa el ingreso global de los recursos en la economía en la forma de salario $w=\alpha Y$ para las personas trabajadoras e ingreso bruto del capital $\hat{\mathrm{w}}=(1-\alpha) Y$. Así,

(50) $W=w+\hat{w}$

Sea $w$ el salario de $L$, igual al costo laboral. De la ecuación (48), resulta:

$$
\text { (51) } 1=F\left[\frac{\alpha}{c}, \frac{1-\alpha}{c}\right]
$$

Sea $\alpha$ $=\frac{\alpha}{c} \therefore \alpha=\not \alpha c$ y $\alpha$ es el indicador de la contribución real del trabajo en el costo medio. Al substituir esa definición en la ecuación (49):

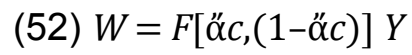

Así, el ingreso de $(L)$ equivale a su costo en términos de $(Y)$ :

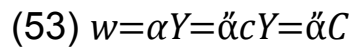


y también el ingreso del capital equivale a su costo:

(54) $\hat{\mathrm{w}}=(1-\alpha) Y=\left(1-\alpha{ }^{\prime \prime} c\right) Y$

Ŵ contiene la ganancia, Ḱ y la depreciación de K en términos de $(Y)$. La participación efectiva del capital en el costo, incluida depreciación, remanente y capital de trabajo, se estima como $(1-\alpha) C=(1, \mathrm{~K}) \mathrm{K}$. El costo efectivo y potencial es $C=\alpha 2 C+(1-\alpha) C$, dadas las tasas de crecimiento. El costo adicional por unidad de trabajo y capital se define como $c_{L}=\dot{c}_{L} \hat{C}$ y $c_{\mathrm{K}}=\hat{c}_{\mathrm{K}} \hat{\mathrm{C}}$, respectivamente, y $\hat{C}_{t}=\hat{C}_{t-1}+c_{L_{t}}+c_{K_{t}}$. El nivel de producción efectiva $(Y)$, o bien estimada $\hat{Y}$, en cada periodo de producción equivale a la ecuación (50):

(55) $W=Y=w+\hat{w}$

El ingreso relativo del trabajo se mide al operar en la ecuación (43) tal que $\frac{\lambda_{L}}{f(\hat{r}, 1) n}=\alpha$ y de multiplicar ambos lados de la expresión por $\lambda$ :

(56) $\gamma_{L}=\alpha \lambda$

Y de la ecuación (45) para el capital:

(57) $\gamma_{\mathrm{K}}=(1-\alpha) \lambda$

Al multiplicar esas dos ecuaciones por $(Y)$ se obtienen los ingresos medios $\bar{y}_{l}, \bar{y}_{\mathrm{K}}$. El equivalente en términos de costos se deriva de la ecuación (32); $\frac{\hat{c}_{L}}{f[(r, 1) n]}=\alpha$ y al multiplicar la expresión por ć resulta:

(58) $\dot{\mathrm{c}}_{L}=\alpha \dot{\alpha}$

y de la ecuación (34) para el capital:

(59) $\dot{\mathrm{c}}_{\mathrm{K}}=(1-\dot{\alpha}) \dot{c}$

De donde se deducen la participación media y total de los recursos en los costos.

\section{Ganancia y tasa de ganancia}

En Cobb y Douglas (1928) se lee que el capital de trabajo 'produce' valor para su propietario, pero aquí no interesan los valores sino el producto físico. Ceteris paribus, por supuesto que en el producto se refleja el valor que 
'produce' ese capital, pero también ese valor podría generar ganancia en la forma $(Y)$. Del desarrollo anterior se puede deducir la ganancia y la tasa de ganancia generada por los recursos de la producción. Sea:

(60) $g=F[(1, \kappa) \mathrm{K} ; L]$

De donde la participación de los recursos es $1=F\left[(1, \kappa) \frac{K}{g} ; \frac{L}{g}\right]$. Sea $\grave{\alpha}=\frac{L}{g}: \therefore 1=F\left(\grave{\alpha}, \frac{\mathrm{K}}{g}\right)$ y $(1-\grave{\alpha})=\frac{\mathrm{K}}{g}$ por lo que $1=F[\grave{\alpha},(1-\grave{\alpha})]$. Al diferenciar la ecuación (60) y reemplazar las definiciones anteriores en el resultado y al simplificar, se obtiene la tasa de ganancia potencial:

(61) $\grave{g}=\grave{\alpha} \mathrm{n} ;(1-\grave{\alpha})[\mathrm{k} \kappa,(1-\grave{\alpha})(1, \mathrm{\kappa}) 2 \delta \mathrm{k}$,

Empero, por la ecuación $(23) \mathrm{r} L=(1, \mathrm{~K}) \mathrm{K}$ y y al insertar en la función (60):

(62) $g=F(\mathrm{r} ; 1) L$

Y por la derivada, dado $(\hat{r}=0)$ se deduce la contribución media del trabajo a la ganancia:

(63) $\dot{\mathrm{g}}_{L}=f(r ; 1) n \grave{\alpha}$

Y la contribución relativa es $\frac{\dot{\mathrm{g}}_{L}}{f(r ; 1) n}=\grave{\alpha}$ y al multiplicar por $(\dot{\mathrm{g}})$ ambos lados de la expresión:

(64) $\pi_{L}=\grave{\alpha} \dot{g}$

Para el capital, se deduce de la ecuación (23) que $L=\frac{1}{r}(1, \mathrm{~K}) \mathrm{K}$ y al insertar en la función (60):

(65) $g=F\left[\left(1, \frac{1}{r}\right)(1, \kappa) \mathrm{K}\right]$

y al diferenciar y al suponer (k,ŕr) $=0$ :

(66) $\dot{\mathrm{g}}_{\mathrm{K}}=f\left[\left(1, \frac{1}{r}\right) k(1-\grave{\alpha})\right]$ 
Para que $\frac{\dot{g}_{k}}{f\left[\left(1, \frac{1}{r}\right) k\right]}=(1-\grave{\alpha})$ y al multiplicar esta expresión por $(\dot{g})$ :

(67) $\pi_{\mathrm{K}}=(1-\grave{\alpha}) \dot{g}$

No obstante $\grave{\alpha}$, se torna difícil de calcular directamente de este componente del modelo. Por suerte, al deducir la ecuación (58) de la (56) se devela (64): $\pi_{L}=\gamma_{L}-\dot{\mathrm{c}}_{L}$ y la contribución media del trabajo es:

(68) $\grave{\alpha} \dot{g}=\alpha \lambda-\alpha \dot{c}$

Y la del capital se obtiene de deducir la ecuación (59) de la (57): $\pi_{\mathrm{K}}=\gamma_{\mathrm{K}}-\dot{\mathrm{C}}_{\mathrm{K}}$ tal que:

(69) $(1-\grave{\alpha}) \dot{g}=(1-\alpha) \lambda-(1-\alpha) \dot{c}$

Al multiplicar esas dos ecuaciones por $(Y)$ se obtienen las contribuciones medias y totales de los recursos a la ganancia.

Sin embargo, g es una tasa efectiva medida sobre la totalidad del costo de producción y no sobre el capital efectivamente consumido: $\check{\mathrm{K}}+\hat{K}+w$. Sea:

$$
\text { (70) } \dot{\mathrm{g}}=\frac{g}{\check{\mathrm{K}}+\dot{K}+w}
$$

donde g es la tasa de ganancia efectiva a cada instante en la economía, equivalente a g, ya que $g=\dot{\mathrm{g}} Y=(\check{\mathrm{K}}+\hat{\mathrm{K}}+w) \dot{\mathrm{g}}$. Empero, ceteris paribus, se supone que el valor $(\mathrm{Y})$ en cada instante equivale al costo de producción más la ganancia global del capital, tal que:

$$
\text { (71) } Y=(\check{\mathrm{K}}+\hat{\mathrm{K}}+w)(1+\dot{\mathrm{g}}) \text {. }
$$

Lo que es lo mismo:

(72) $Y=\check{K}+\check{K}+w+g$

la cual es idéntica a la ecuación (55), dado que el ingreso del capital de compone de $\hat{\mathrm{w}}=\check{\mathrm{K}}+\hat{\mathrm{K}}+g$.

\section{Consumo de capital o depreciación y el remanente}


Se puede obtener el consumo de capital fijo o depreciado Ǩ y el capital fijo remanente қ al deducir de la producción el costo laboral y la ganancia. De la ecuación (72):

(73) $\check{\mathrm{K}}=Y-(\hat{\mathrm{K}}+w+g)$

Por la distribución de esos capitales según su acumulación a cada instan-

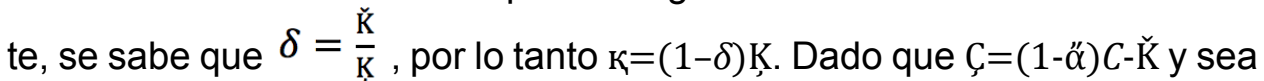
$c ̧=(1-\alpha) C$ para que $C \zeta=c ̧-\check{K}$, lo equivale a la suma de remanentes de capital fijo к y capital laboral $L_{\mathrm{K}}$. Este último surge de $L_{\mathrm{K}}=\left({ }^{\prime} \alpha-\alpha\right) C$ y al deducir este resultado de $C$ se obtiene el capital fijo remanente a cada instante. De aquí se puede estimar el capital fijo adicional к como resultado del proceso de acumulación de un instante a otro o la acumulación efectiva a cada instante neta de la depreciación:

(74) $\kappa_{t}=\mathrm{K}_{t}-\mathrm{K}_{t-1} \cdot$

\section{CONCLUSIONES, IMPLICACIONES Y POSIBLES LÍNEAS DE INVESTIGACIÓN}

Esta investigación se origina en la sugerencia de Cobb y Douglas (1928) acerca de la necesidad de medir la contribución relativa de los recursos a la producción a cada instante y de eliminar el tiempo como variable. Se indicó, también, que Villalobos (2019) contempla tales sugerencias y que en Solow (1957), Arrow et al. (1961) y en la Contabilidad del crecimiento se realizó un esfuerzo de distinta naturaleza. Esta investigación se planteó, con base en supuestos críticos rigurosos, una contribución a la teoría del crecimiento económico a partir del análisis del costo de producción. Se introduce la viabilidad tecnológica como capacidad de los recursos capital fijo y trabajo para transformar materias primas e insumos y transferir el valor global de los recursos al producto. Ello permitió elaborar nuevas fórmulas con las cuales medir la contribución y participación relativa, media y total de los recursos en los costos de producción y en el producto.

Un resultado fundamental, que difiere de los aportes teóricos y empíricos existentes, es la factibilidad de la medición de los valores $\forall\{\alpha, \alpha, \alpha, \alpha, \alpha:(0,1)\}$ a cada instante -por ejemplo, anual. Asimismo, se propone una medida de la elasticidad de depreciación y elasticidad de composición de los recursos en general y del capital-trabajo en particular. Se plantea la posibilidad de 
cuantificar la depreciación, el remanente del capital, el residuo y la ganancia en términos del producto. Los procedimientos derivados del supuesto crítico de variabilidad en las condiciones fundamentales de la economía, con que se formula este aporte a la teoría del crecimiento económico, permite medir la equivalencia entre el costo y el ingreso medio de los recursos. Lo anterior es pertinente con la sentencia de Harrod (1939), quien arguyó que una vez que la mente se acostumbra a pensar en términos de tendencias de crecimiento, los viejos problemas de la formulación estática parecen rancios, llanos e infructuosos. En función de esa sentencia crucial y de los resultados de esta investigación, se podría concluir que "los procesos de distribución son...modelados por los procesos de producción de valor" (Cobb \& Douglas, 1928, pp. 139-140).

La simplificación teórica del modelo propuesto no es exclusiva de la desagregación de las variables que lo componen; entre otras, impuestos, precios, tasa de interés y tipo de cambio. A efectos de validar tentativamente su rigurosidad y su capacidad analítica y explicativa, se evalúa el modelo con base en los índices de producción, capital y trabajo generados por Cobb y Douglas (1928), cuyos resultados se muestran en el anexo de esta investigación. En el contexto de las limitaciones mencionadas, entre las tareas pendientes está aplicar este modelo a una serie de datos de periodos más recientes, con objeto de validar su robustez y contrastar los resultados con otros modelos. Ese ejercicio podrá realizarse tanto al nivel de empresa o industria como de las economías. Esta investigación podría ser útil a efectos académicos, de donde podría además resultar fortalecido, y su extensión empírica pertinente podrá generar implicaciones relevantes para las políticas de gobierno y de las empresas.

\section{REFERENCIAS}

Abramovitz, M. (1962). Economic growth in the United States. The American Economic Review , 52 (4), 762-782.

Abramovitz, M. (1956). Resource and Output Trends in the United States since 1870. (Vol. 46). Pittsburgh: American Economic Review Papers and Proceedings. 
Arrow, J. K., Chenery, B. H., Minhas, S. B., \& Solow, M. R. (1961). Capital-Labor substitution and economic efficiency. The Review of Economics and Statistics , 43 (3), 225-250.

Barro, R. J., \& Sala-i-Martin, X. (2004). Economic growth (Second ed.). London: The MIT Press.

Cobb, W. C., \& Douglas, H. P. (1928). A Theory of Production. The American Economic Review , 18 (1), 139-165.

Crafts, N. (2009). Solow and growth accounting:. History of Political Economy 41 (41), 200-220.

Domar, E. D. (1946). Capital Expansion, Rate of Growth, and Employment. Econometrica , 14 (02), 137-147.

Domar, E. D. (1953). Depreciation, Replacement and Growth. The Economic Journal , 63 (249), 1-32.

Harrod, R. F. (1939). An essay in dynamic theory. The Economic Journal , 49 (193), 14-33.

Hulten, C. R. (2009, 9). NBER WORKING PAPER SERIES. (N. B. RESEARCH, Ed.) Retrieved 7 23, 2019, from NBER WORKING PAPER SERIES: http://www.nber.org/papers/w15341

Keynes, J. M. (1964). The general theory of employment, interest, and money (First ed.). United States of America: A Harvest/HBJ Book.

Piketty, T. (2014). Capital in the Twenty-First Cenury. London: The Belknap Press of Harvard University Press.

Solow, M. R. (1956). A contribution to the theory of economic growth. The Quarterly Journal of Economics , 70 (1), 65-94.

Solow, M. R. (1957). Technical Change and the Aggregate Production Function. The Review of Economics and Statistics , 39 (3), 312-320.

Swan, T. (1956). Economic growth and capital accumulation. Economic Record, 334-361.

Villalobos, D. (2019). Crecimiento económico: Convergencia y divergencia. Economía y Sociedad , 24 (55), 1-30. Doi: http://dx.doi.org/10.15359/ eys.23-55.3. 


\section{ANEXO}

\section{Una evaluación preliminar del modelo}

Dentro de los límites posibles, este anexo pretende valuar el modelo aquí propuesto a partir de los indicadores de producción, capital y trabajo elaborados por Cobb y Douglas (1928) para la manufactura de los Estados Unidos de América durante 1899-1922. Dado que esta investigación se parte del costo de producción, en esos datos se desconoce $K$ Ḱ tal que $(\kappa=0)$, lo que induce una limitación en la evaluación del modelo propuesto. Se asume $(Y)$ un resultado del proceso de producción a cada instante según la dinámica de $(K, L)$, por consiguiente $(Y)$ contiene $(K, K, L)$ transformados y transferidos en cada instante por el proceso de producción, más la ganancia. En tal caso, Ḱ aparecerá como componente de la contribución y participación de Ķ en $(C$, $Y, g)$ según $(0<\alpha, \alpha, \alpha, \alpha, \alpha<1)$. Los índices analizados se asumen como valores reales, según criterio de año base con que fueron computados. Esto implica que $(K, K, L)$ contribuyen y participan en $(C, g)$ en la forma de $(Y)$, lo que destaca la distribución de $(Y)$. Si bien el análisis propuesto es completo, solo se describen los resultados que a nuestro juicio son relevantes.

La Tabla 1 registra los valores para cada variable fundamental del modelo, obtenidos con las fórmulas que lo definen. Entre otros resultados, se destaca que $(0<\alpha, \grave{\alpha}, \dot{\alpha}, \ddot{\alpha}<1)$ y varían dentro de ese rango a cada instante, tal como lo sugirieron Cobb y Douglas y acorde con los supuestos críticos del modelo. La elasticidad de composición de los recursos $(v)$ y del capital-trabajo $(\mu)$ son mayores que cero: $[(v, \mu)>0]$. La Figura 1 ilustra la tendencia creciente de la composición de los recursos ( $r$ ) durante 1899-1922 y como consecuencia en su elasticidad, induciendo desplazamiento trabajo en los años 1918-1922, caracterizados por recesión y depresión de la economía. La Figura 2 contrasta la tendencia $(r)$ y la oscilación registrada por $\mu$ durante 1899-1922. Las tasas de crecimiento del costo y del producto muestran una relativa afinidad en el rango $\mu:(0,3)$; sin embargo, el producto declina oscilante en ese período conforme $(\mu>0)$ (Figura 3 ).

Las tasas efectivas de variación anual de los valores reales del capital y del trabajo (Tabla 1) y la determinación del valor de à a cada instante, permitieron la distribución del costo global según la contribución relativa, media y total de los recursos (Tabla 2). La participación total de de cada recurso en los costos globales se mide como costo-ingreso del trabajo $(w)$ y del capital $(\hat{w})$ por medio de $\alpha$, que en conjunto equivalen al valor del producto anual. 
Durante 1899-1922, $(w)$ muestra una tendencia a crecer mientras que $(\hat{w})$ declina conforme $(\mu>0)$. Se denota mayor convergencia costo-ingreso entre esos recursos en el rango $(1 \cong \mu<2)$ (Figura 4$)$, en consonancia con las tasas de crecimiento del costo global y del producto (Figura 3 ). En periodos en que predomina relativa prosperidad de los negocios, se observa $(\mu \geq 1)$.

Con base en los valores de $(\alpha, \lambda)$ (Tabla 1$)$, el modelo logra medir la contribución relativa, media y total del capital y del trabajo, así como su contribución conjunta, al crecimiento del producto (Tabla 3). Esa contribución de los recursos diverge en cada instante en razón de las variaciones en $\alpha$. La distribución del producto en la forma de ingresos $(w, \hat{w})$ equivale a la contribución en los costos en razón de $\alpha$ (Tabla 2, Figura 4). Además, en $[\mu:(0,1)] ;(w<\hat{w})$; no obstante $(w>\hat{w})$ en períodos de prosperidad. La contribución relativa del trabajo al producto diverge respecto del capital al nivel de $(\mu<4)$ y la del trabajo denota mayor en la volatilidad; al nivel de $(\mu$ $\geq 2.5$ ) ambas contribuciones declinan, pero la del trabajo es más pronunciada. Empero, la contribución relativa de esos recursos al costo es semejante cuando $(\mu<4)$ (Figuras 5 y 6$)$. La conjunción de tales tendencias se revela congruente en la Figura 3.

Estos resultados podrían haber influido en la tasa de ganancia según contribución de los recursos (Tabla 4, Figuras 7 y 8). Durante 1899-1922, esa tasa fue creciente y oscilante conforme $(\mu>0)$ y la contribución del trabajo supera la del capital a cada nivel de $(\mu)$. Esa dinámica se refleja en Ǩ, la cual pudo ser mayor en periodos de prosperidad, mientras el capital fijo remanente y la acumulación $\kappa$ disminuyeron (Tabla 5, Figura 9). La tasa de depreciación se mostró alta en $(\mu<2)$, y las tasas de reposición y acumulación tienden a converger; esas tendencias responden a condiciones de relativa prosperidad económica. Cuando $(\mu \geq 2)$ todas esas tasas se deprimen negativamente, con mayor fuerza la de depreciación. Estos resultados sugieren influencia de los ciclos de los negocios, afectados por la actividad bélica en torno a la I Guerra Mundial. En el año 2015 se redujo fuertemente el consumo de capital $(\mathrm{k}=-0.4519)$ y $(\mu=2.6869 ;$ '́ $=-0.0486$; $\mathrm{k}=-0.0122$ ) cuando la manufactura se recuperaba de la depresión del año 1914 en víspera de la I Guerra Mundial, una tasa de empleo decreciente, alta inversión en capital y una caída en la producción: $(n=-0.03 ; k=0.05$; $\lambda=-0.08$ ) (Tabla 1). En el año 1916, probablemente a causa de la prosperidad de los negocios $(\mu=0.6617 ; \kappa=0.2123 ; \mathrm{k}=0.3595 ; \mathrm{k}=1.3067)$ (Tabla 5) y $(n=0.18 ; k=0.12 ; \lambda=0.19)$ (Tabla 1$)$. 
Durante 1916-1920, con elevadas tasas de empleo e inversión en capital, alta tasa de crecimiento de la depreciación en el año $1919(57 \%)$ y la más fuerte acumulación de capital $(27 \%)$. No obstante, la actividad bélica y momentos de recesión económica deprimieron la producción. La alta inversión en capital en ese año no logró realizarse en los siguientes; $[\mu>2 ;(\hat{\kappa}, \mathrm{k})<0$; $\mathrm{k}=(0,1)$ ] y el empleo es muy bajo e incluso negativo (Tabla 1 y 5 , Figura 9). En resumen, el capital desempleado es extremadamente importante, porque su presencia inhibe nueva inversión (Domar, 1946).

\section{Tabla 1}

\section{Modelo de costo efectivo en el crecimiento de la manufactura en Estados Unidos (1899-1922)}

\begin{tabular}{|c|c|c|c|c|c|c|c|c|c|c|c|c|c|c|c|c|c|c|c|c|}
\hline Año & $\mathrm{Y}$ & $L$ & $\mathrm{~K}$ & C & $\lambda$ & $n$ & $k$ & ć & c & $\hat{\mathbf{r}}$ & $\hat{\mathbf{c}}$ & č & $\ddot{\mathrm{r}}$ & $\mu$ & $\alpha$ & $\dot{\alpha}$ & $\ddot{\alpha}$ & $v$ & $\dot{\mathrm{r}}$ & Ciclo de negocios \\
\hline 1899 & 100 & 100 & 100 & 200 & - & - & - & - & 2.00 & 1.00 & - & - & - & - & - & - & - & - & - & Prosperity \\
\hline 1900 & 101 & 105 & 107 & 212 & 0.01 & 0.05 & 0.07 & 0.06 & 2.10 & 1.02 & 2.02 & 1.98 & 0.02 & 1.40 & 0.58 & 0.58 & 0.28 & 1.40 & 0.01 & Prosperity; brief recession \\
\hline 1901 & 112 & 110 & 114 & 224 & 0.11 & 0.05 & 0.07 & 0.06 & 2.00 & 1.04 & 2.04 & 1.96 & 0.02 & 1.37 & 0.57 & 0.57 & 0.29 & 1.37 & 0.01 & Prosperity \\
\hline 1902 & 122 & 118 & 122 & 240 & 0.09 & 0.07 & 0.07 & 0.07 & 1.97 & 1.03 & 2.03 & 1.97 & 0.00 & 0.96 & 0.48 & 0.48 & 0.25 & 0.96 & 0.00 & Prosperity \\
\hline 1903 & 124 & 123 & 131 & 254 & 0.02 & 0.04 & 0.07 & 0.06 & 2.05 & 1.07 & 2.07 & 1.94 & 0.03 & 1.74 & 0.62 & 0.62 & 0.30 & 1.74 & 0.02 & Prosperity; recession \\
\hline 1904 & 122 & 116 & 138 & 254 & -0.02 & -0.06 & 0.05 & 0.00 & 2.08 & 1.19 & 2.19 & 1.84 & 0.11 & 0.94 & 0.44 & 0.44 & 0.21 & 0.94 & 0.00 & Mild depression; revival \\
\hline 1905 & 143 & 125 & 149 & 274 & 0.17 & 0.08 & 0.08 & 0.08 & 1.92 & 1.19 & 2.19 & 1.84 & 0.00 & 1.03 & 0.46 & 0.46 & 0.24 & 1.03 & 0.01 & Prosperity \\
\hline 1906 & 152 & 133 & 163 & 296 & 0.06 & 0.06 & 0.09 & 0.08 & 1.95 & 1.23 & 2.23 & 1.82 & 0.03 & 1.47 & 0.55 & 0.55 & 0.28 & 1.47 & 0.03 & Prosperity \\
\hline 1907 & 151 & 138 & 176 & 314 & -0.01 & 0.04 & 0.08 & 0.06 & 2.08 & 1.28 & 2.28 & 1.78 & 0.04 & 2.12 & 0.62 & 0.62 & 0.30 & 2.12 & 0.04 & Prosperity; panic; recession; depression \\
\hline 1908 & 156 & 121 & 185 & 306 & 0.03 & -0.12 & 0.05 & -0.03 & 1.96 & 1.53 & 2.53 & 1.65 & 0.17 & 0.42 & 0.21 & 0.21 & 0.11 & 0.42 & 0.01 & Depression \\
\hline 1909 & 125 & 140 & 198 & 338 & -0.20 & 0.16 & 0.07 & 0.10 & 2.70 & 1.41 & 2.41 & 1.71 & -0.09 & 0.45 & 0.24 & 0.24 & 0.09 & 0.45 & -0.01 & Revival; Mild prosperity \\
\hline 1910 & 159 & 144 & 208 & 352 & 0.27 & 0.03 & 0.05 & 0.04 & 2.21 & 1.44 & 2.44 & 1.69 & 0.02 & 1.77 & 0.55 & 0.55 & 0.25 & 1.77 & 0.02 & Recession \\
\hline 1911 & 153 & 145 & 216 & 361 & -0.04 & 0.01 & 0.04 & 0.03 & 2.36 & 1.49 & 2.49 & 1.67 & 0.03 & 5.54 & 0.79 & 0.79 & 0.33 & 5.54 & 0.04 & Mild depression \\
\hline 1912 & 177 & 152 & 226 & 378 & 0.16 & 0.05 & 0.05 & 0.05 & 2.14 & 1.49 & 2.49 & 1.67 & 0.00 & 0.96 & 0.39 & 0.39 & 0.18 & 0.96 & 0.01 & Revival; prosperity \\
\hline 1913 & 184 & 154 & 236 & 390 & 0.04 & 0.01 & 0.04 & 0.03 & 2.12 & 1.53 & 2.53 & 1.65 & 0.03 & 3.36 & & & 0.32 & 3.36 & 0.04 & Prosperity; recession \\
\hline 1914 & 169 & 149 & 244 & 393 & -0.08 & -0.03 & 0.03 & 0.01 & 2.33 & 1.64 & 2.64 & 1.61 & 0.07 & & & & 0.17 & 1.04 & -0.01 & \\
\hline 1915 & 189 & 154 & 266 & 420 & 0.12 & 0.03 & 0.09 & 0.07 & 2.22 & 1.73 & 2.73 & 1.58 & 0.06 & 2.69 & 0.6 & & 0.2 & 2.69 & 0.07 & Revir \\
\hline 1916 & 225 & 182 & 298 & 480 & 0.19 & 0.18 & 0.12 & 0.14 & 2.13 & 1.64 & 2.64 & 1.61 & -0.06 & 0.66 & 0.29 & 0.29 & 0.13 & 0.66 & 0.00 & Prosperity \\
\hline 1917 & 227 & 196 & 335 & 531 & 0.01 & 0.08 & 0.12 & 0.11 & 2.34 & 1.71 & 2.71 & 1.59 & 0.05 & 1.61 & 0.49 & 0.49 & 0.21 & 1.61 & 0.07 & Prosperity; war activity \\
\hline 1918 & 223 & 200 & 366 & 566 & -0.02 & 0.02 & 0.09 & 0.07 & 2.54 & 1.83 & 2.83 & 1.55 & 0.07 & 4.53 & 0.71 & 0.71 & 0.28 & 4.53 & 0.11 & War activity; recession \\
\hline 1919 & 218 & 193 & 387 & 580 & -0.02 & -0.04 & 0.06 & 0.02 & 2.66 & 2.01 & 3.01 & 1.50 & 0.09 & 1.64 & 0.45 & 0.45 & 0.17 & 1.64 & -0.04 & Revival; prosperity \\
\hline 1920 & 231 & 193 & 407 & 600 & 0.06 & 0.00 & 0.05 & 0.03 & 2.60 & 2.11 & 3.11 & 1.47 & 0.05 & 2.11 & 0.50 & 0.50 & $0.19^{\prime \prime}$ & 2.11 & 0.00 & Prosperity; recession; depression \\
\hline 1921 & 179 & 147 & 417 & 564 & -0.23 & -0.24 & 0.02 & -0.06 & 3.15 & 2.84 & 3.84 & 1.35 & 0.26 & 0.10 & 0.04 & 0.04 & 0.01 & 0.10 & 0.01 & Depression \\
\hline 1922 & 240 & 161 & 431 & 592 & 0.34 & 0.10 & 0.03 & 0.05 & 2.47 & 2.68 & 3.68 & 1.37 & -0.06 & 0.35 & 0.12 & 0.12 & 0.05 & 0.35 & 0.00 & Revival; prosperity \\
\hline
\end{tabular}

Nota. Tabla basada en el modelo propuesto y datos de Cobb y Douglas (1928).

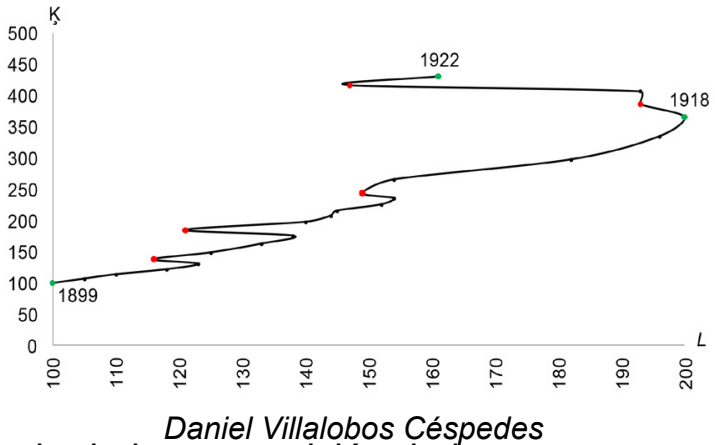

Figura 1. Tendencia de la composjición de los recursos en la manufactura 
de Estados Unidos (1899-1922). Con base en Tabla 1.

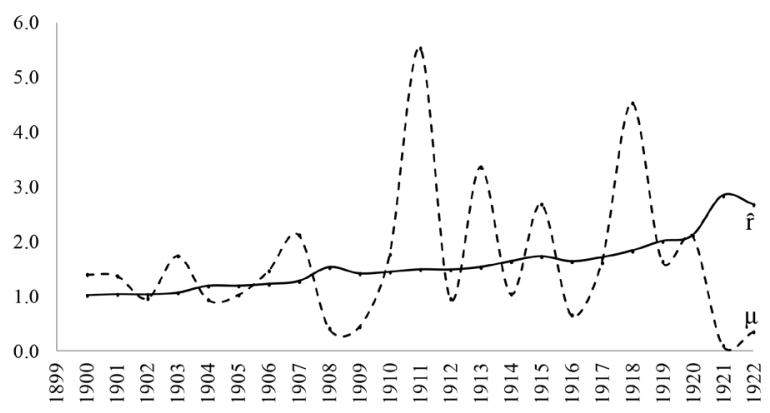

Figura 2. Tendencia de la composición de los recursos en la manufactura de Estados Unidos (1899-1922) según $\mu$. Con base en Tabla 1.

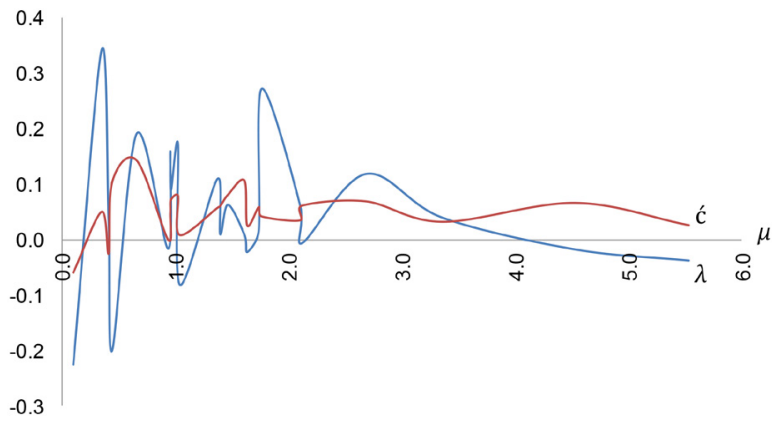

Figura 3. Tendencia de la tasa de crecimiento del costo y la producción estimados para la manufactura de Estados Unidos (1899-1922) respecto de $\mu$. Con base en Tabla 1.

\section{Tabla 2}

Crecimiento estimado del costo efectivo, contribución de los recursos y costo del trabajo y del capital para la manufactura de Estados Unidos (1899-1922) 


\begin{tabular}{|c|c|c|c|c|c|c|c|c|c|c|c|}
\hline Año & $\dot{\mathrm{C}}_{\mathrm{L}}$ & $\dot{C}_{\mathrm{K}}$ & ć & C & $c_{\mathrm{L}}$ & $C_{\underline{K}}$ & ćC & $w$ & $\hat{\mathrm{w}}$ & ç & Ciclo de negocios \\
\hline 1899 & - & - & - & 200 & - & - & - & - & - & - & Prosperity \\
\hline 1900 & 0.0347 & 0.0253 & 0.0600 & 212 & 6.9 & 5.1 & 12 & 58 & 43 & 154 & Prosperity; brief recession \\
\hline 1901 & 0.0323 & 0.0243 & 0.0566 & 224 & 6.8 & 5.2 & 12 & 64 & 48 & 160 & Prosperity \\
\hline 1902 & 0.0345 & 0.0369 & 0.0714 & 240 & 7.7 & 8.3 & 16 & 59 & 63 & 181 & Prosperity \\
\hline 1903 & 0.0362 & 0.0221 & 0.0583 & 254 & 8.7 & 5.3 & 14 & 77 & 47 & 177 & Prosperity; recession \\
\hline 1904 & -0.0534 & 0.0534 & 0.0000 & 254 & -13.6 & 13.6 & 0 & 54 & 68 & 200 & Mild depression; revival \\
\hline 1905 & 0.0364 & 0.0423 & 0.0787 & 274 & 9.3 & 10.7 & 20 & 66 & 77 & 208 & Prosperity \\
\hline 1906 & 0.0438 & 0.0365 & 0.0803 & 296 & 12.0 & 10.0 & 22 & 83 & 69 & 213 & Prosperity \\
\hline 1907 & 0.0380 & 0.0228 & 0.0608 & 314 & 11.2 & 6.8 & 18 & 94 & 57 & 220 & Prosperity; panic; recession; depression \\
\hline 1908 & -0.0054 & -0.0200 & -0.0255 & 306 & -1.7 & -6.3 & -8 & 33 & 123 & 273 & Depression \\
\hline 1909 & 0.0251 & 0.0794 & 0.1046 & 338 & 7.7 & 24.3 & 32 & 30 & 95 & 308 & Revival; Mild prosperity \\
\hline 1910 & 0.0228 & 0.0186 & 0.0414 & 352 & 7.7 & 6.3 & 14 & 88 & 71 & 265 & Recession \\
\hline 1911 & 0.0201 & 0.0054 & 0.0256 & 361 & 7.1 & 1.9 & 9 & 121 & 32 & 240 & Mild depression \\
\hline 1912 & 0.0185 & 0.0286 & 0.0471 & 378 & 6.7 & 10.3 & 17 & 69 & 108 & 309 & Revival; prosperity \\
\hline 1913 & 0.0218 & 0.0099 & 0.0317 & 390 & 8.2 & 3.8 & 12 & 126 & 58 & 264 & Prosperity; recession \\
\hline 1914 & 0.0030 & 0.0047 & 0.0077 & 393 & 1.2 & 1.8 & 3 & 66 & 103 & 327 & Depression \\
\hline 1915 & 0.0418 & 0.0269 & 0.0687 & 420 & 16.4 & 10.6 & 27 & 115 & 74 & 305 & Revival; prosperity \\
\hline 1916 & 0.0411 & 0.1017 & 0.1429 & 480 & 17.3 & 42.7 & 60 & 65 & 160 & 415 & Prosperity \\
\hline 1917 & 0.0516 & 0.0546 & 0.1063 & 531 & 24.8 & 26.2 & 51 & 110 & 117 & 421 & Prosperity; war activity \\
\hline 1918 & 0.0470 & 0.0190 & 0.0659 & 566 & 24.9 & 10.1 & 35 & 159 & 64 & 407 & War activity; recession \\
\hline 1919 & 0.0111 & 0.0136 & 0.0247 & 580 & 6.3 & 7.7 & 14 & 98 & 120 & 482 & Revival; prosperity \\
\hline 1920 & 0.0172 & 0.0172 & 0.0345 & 600 & 10.0 & 10.0 & 20 & 116 & 116 & 485 & Prosperity; recession; depression \\
\hline 1921 & -0.0021 & -0.0579 & -0.0600 & 564 & -1.3 & -34.7 & -36 & 6 & 173 & 558 & Depression \\
\hline 1922 & 0.0058 & 0.0439 & 0.0496 & 592 & 3.3 & 24.7 & 28 & 28 & 212 & 564 & Revival; prosperity \\
\hline
\end{tabular}

Nota. Con base en Tabla 1.

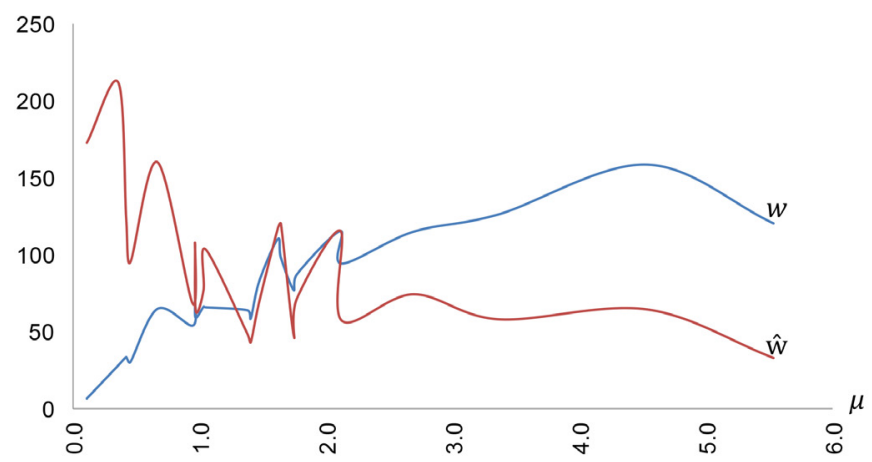

Figura 4. Costo efectivo anual del trabajo y el capital estimados para la manufactura de Estados Unidos (1899-1922) acorde con $\mu$. Con base en Tabla 2.

Tabla 3

Crecimiento efectivo de la producción, participación de los recursos e ingreso del trabajo y del capital en la manufactura de Estados Unidos (1899-1922)

Daniel Villalobos Céspedes 


\begin{tabular}{lcccccccccl}
\hline Año & $\gamma_{L}$ & $\gamma_{K}$ & $\lambda$ & $Y$ & $\breve{y}_{L}$ & $\breve{y}_{K}$ & $\lambda Y$ & $w$ & $\hat{w}$ & \multicolumn{1}{c}{ Ciclo de negocios } \\
\hline 1899 & - & - & - & 100 & - & - & - & - & - & Prosperity \\
1900 & 0.006 & 0.004 & 0.010 & 101 & 0.6 & 0.4 & 1 & 58 & 43 & Prosperity; brief recession \\
1901 & 0.062 & 0.047 & 0.109 & 112 & 6.3 & 4.7 & 11 & 64 & 48 & Prosperity \\
1902 & 0.043 & 0.046 & 0.089 & 122 & 4.8 & 5.2 & 10 & 59 & 63 & Prosperity \\
1903 & 0.010 & 0.006 & 0.016 & 124 & 1.2 & 0.8 & 2 & 77 & 47 & Prosperity; recession \\
1904 & -0.007 & -0.009 & -0.016 & 122 & -0.9 & -1.1 & -2 & 54 & 68 & Mild depression; revival \\
1905 & 0.080 & 0.092 & 0.172 & 143 & 9.7 & 11.3 & 21 & 66 & 77 & Prosperity \\
1906 & 0.034 & 0.029 & 0.063 & 152 & 4.9 & 4.1 & 9 & 83 & 69 & Prosperity \\
1907 & -0.004 & -0.002 & -0.007 & 151 & -0.6 & -0.4 & -1 & 94 & 57 & Prosperity; panic; recession; depression \\
1908 & 0.007 & 0.026 & 0.033 & 156 & 1.1 & 3.9 & 5 & 33 & 123 Depression \\
1909 & -0.048 & -0.151 & -0.199 & 125 & -7.5 & -23.5 & -31 & 30 & 95 & Revival; Mild prosperity \\
1910 & 0.150 & 0.122 & 0.272 & 159 & 18.7 & 15.3 & 34 & 88 & 71 & Recession \\
1911 & -0.030 & -0.008 & -0.038 & 153 & -4.7 & -1.3 & -6 & 121 & 32 & Mild depression \\
1912 & 0.062 & 0.095 & 0.157 & 177 & 9.4 & 14.6 & 24 & 69 & 108 Revival; prosperity \\
1913 & 0.027 & 0.012 & 0.040 & 184 & 4.8 & 2.2 & 7 & 126 & 58 Prosperity; recession \\
1914 & -0.032 & -0.050 & -0.082 & 169 & -5.8 & -9.2 & -15 & 66 & 103 Depression \\
1915 & 0.072 & 0.046 & 0.118 & 189 & 12.2 & 7.8 & 20 & 115 & 74 Revival; prosperity \\
1916 & 0.055 & 0.136 & 0.190 & 225 & 10.4 & 25.6 & 36 & 65 & 160 Prosperity \\
1917 & 0.004 & 0.005 & 0.009 & 227 & 1.0 & 1.0 & 2 & 110 & 117 Prosperity; war activity \\
1918 & -0.013 & -0.005 & -0.018 & 223 & -2.8 & -1.2 & -4 & 159 & 64 War activity; recession \\
1919 & -0.010 & -0.012 & -0.022 & 218 & -2.2 & -2.8 & -5 & 98 & 120 Revival; prosperity \\
1920 & 0.030 & 0.030 & 0.060 & 231 & 6.5 & 6.5 & 13 & 116 & 116 Prosperity; recession; depression \\
1921 & -0.008 & -0.217 & -0.225 & 179 & -1.8 & -50.2 & -52 & 6 & 173 Depression \\
1922 & 0.040 & 0.301 & 0.341 & 240 & 7.1 & 53.9 & 61 & 28 & 212 Revival; prosperity \\
\hline & & & & & & & & &
\end{tabular}

Nota. Con base en Tabla 1.

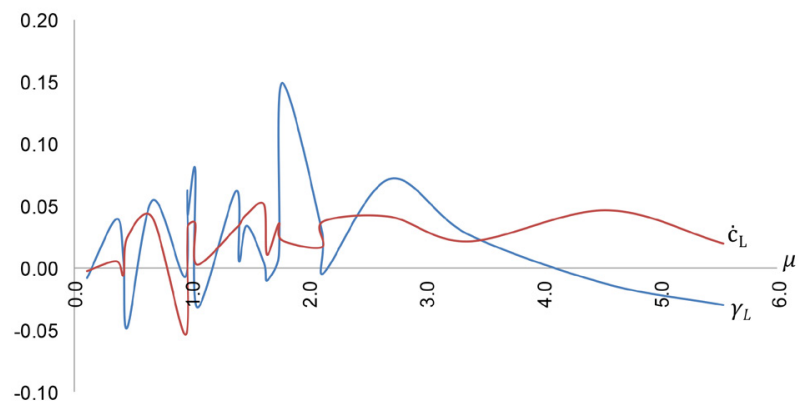

Figura 5. Contribución relativa del trabajo en el costo y en la producción en la manufactura de Estados Unidos (1899-1922) acorde con $\mu$. Con base en Tablas 2 y 3. 


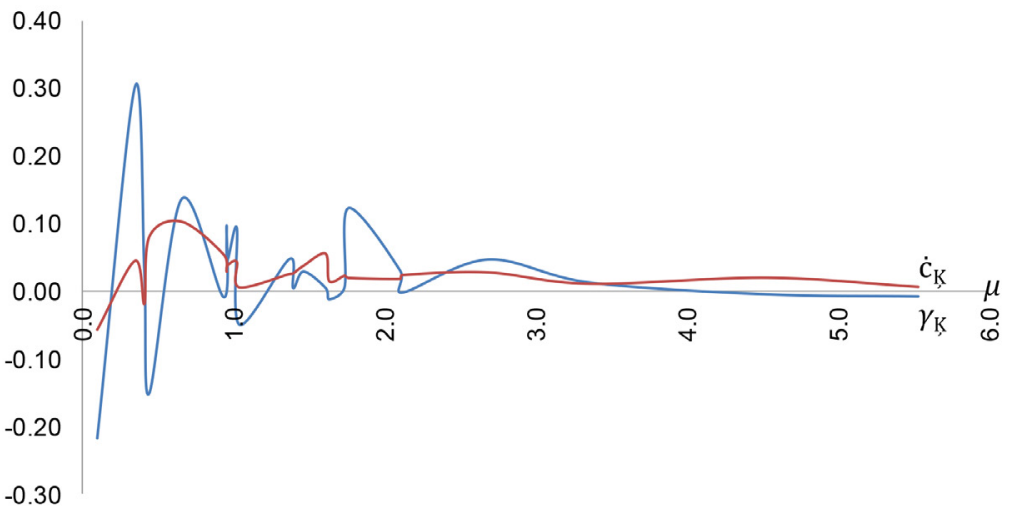

Figura 6. Contribución relativa del capital en el costo y en la producción en la manufactura de Estados Unidos (1899-1922) acorde con $\mu$. Con base en Tablas 2 y 3.

Tabla 4

Contribución efectiva estimada de los recursos en la ganancia generada en la manufactura de estados Unidos (1899-1922)

\begin{tabular}{|c|c|c|c|c|c|c|c|c|c|c|}
\hline Año & $\pi_{L}$ & $\pi_{\underline{k}}$ & ǵ & $\mathrm{g}_{\mathrm{L}}$ & $g_{K}$ & $\mathrm{~g}$ & К̌ & C & $\dot{\mathrm{g}}$ & Ciclo de negocios \\
\hline 1899 & - & - & - & - & - & - & - & - & - & 100 Prosperity \\
\hline 1900 & -0.03 & -0.02 & -0.05 & -2.89 & -2.11 & -5.00 & 48 & 106 & -0.05 & 101 Prosperity; brief recession \\
\hline 1901 & 0.03 & 0.02 & 0.05 & 3.01 & 2.27 & 5.28 & 43 & 117 & 0.05 & 112 Prosperity \\
\hline 1902 & 0.01 & 0.01 & 0.02 & 0.97 & 1.03 & 2.00 & 61 & 120 & 0.02 & 122 Prosperity \\
\hline 1903 & -0.03 & -0.02 & -0.04 & -3.17 & -1.94 & -5.12 & 52 & 125 & -0.04 & 124 Prosperity; recession \\
\hline 1904 & 0.05 & -0.06 & -0.02 & 5.74 & -7.74 & -2.00 & 70 & 130 & -0.02 & 122 Mild depression; revival \\
\hline 1905 & 0.04 & 0.05 & 0.09 & 5.27 & 6.12 & 11.39 & 65 & 142 & 0.09 & 143 Prosperity \\
\hline 1906 & -0.01 & -0.01 & -0.02 & -1.35 & -1.13 & -2.48 & 72 & 142 & -0.02 & 152 Prosperity \\
\hline 1907 & -0.04 & -0.03 & -0.07 & -6.40 & -3.85 & -10.24 & 67 & 153 & -0.06 & 151 Prosperity; panic; recession; depression \\
\hline 1908 & 0.01 & 0.05 & 0.06 & 1.89 & 6.96 & 8.85 & 114 & 159 & 0.06 & 156 Depression \\
\hline 1909 & -0.07 & -0.23 & -0.30 & -11.37 & -35.94 & -47.31 & 142 & 166 & -0.27 & 125 Revival; Mild prosperity \\
\hline 1910 & 0.13 & 0.10 & 0.23 & 15.86 & 12.96 & 28.82 & 43 & 222 & 0.22 & 159 Recession \\
\hline 1911 & -0.05 & -0.01 & -0.06 & -7.93 & -2.13 & -10.07 & 42 & 198 & -0.06 & 153 Mild depression \\
\hline 1912 & 0.04 & 0.07 & 0.11 & 6.59 & 10.21 & 16.80 & 91 & 218 & 0.10 & 177 Revival; prosperity \\
\hline 1913 & 0.01 & 0.00 & 0.01 & 0.95 & 0.43 & 1.38 & 56 & 207 & 0.01 & 184 Prosperity; recession \\
\hline 1914 & -0.03 & -0.05 & -0.09 & -6.39 & -10.02 & -16.42 & 120 & 208 & -0.09 & 169 Depression \\
\hline 1915 & 0.03 & 0.02 & 0.05 & 5.11 & 3.28 & 8.39 & 66 & 239 & 0.05 & 189 Revival; prosperity \\
\hline 1916 & 0.01 & 0.03 & 0.05 & 2.59 & 6.41 & 9.00 & 151 & 264 & 0.04 & 225 Prosperity \\
\hline 1917 & -0.05 & -0.05 & -0.10 & -10.64 & -11.27 & -21.91 & 139 & 282 & -0.09 & 227 Prosperity; war activity \\
\hline 1918 & -0.06 & -0.02 & -0.08 & -13.51 & -5.45 & -18.96 & 83 & 324 & -0.08 & 223 War activity; recession \\
\hline 1919 & -0.02 & -0.03 & -0.05 & -4.73 & -5.79 & -10.52 & 130 & 351 & -0.05 & 218 Revival; prosperity \\
\hline 1920 & 0.01 & 0.01 & 0.03 & 2.74 & 2.74 & 5.48 & 110 & 374 & 0.02 & 231 Prosperity; recession; depression \\
\hline 1921 & -0.01 & -0.16 & -0.17 & -1.34 & -36.80 & -38.14 & 211 & 347 & -0.18 & 179 Depression \\
\hline 1922 & 0.03 & 0.26 & 0.29 & 6.06 & 46.05 & 52.11 & 160 & 404 & 0.28 & 240 Revival; prosperity \\
\hline
\end{tabular}

Nota. Con base en Tabla 1.

Daniel Villalobos Céspedes 


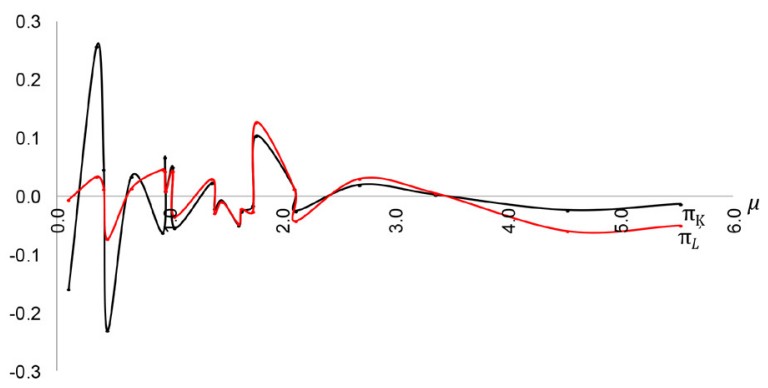

Figura 7. Contribución relativa estimada de los recursos en la ganancia para la manufactura de Estados Unidos (1899-1922) según $\mu$. Con base en tabla 4.

\section{Tabla 5}

Capital fijo: Estimados del consumo o depreciación, remanente y formación efectiva (1899-1922)

\begin{tabular}{|c|c|c|c|c|c|c|c|c|c|c|c|c|c|c|c|}
\hline Año & $\mathrm{K}$ & $\delta$ & $1-\delta$ & Ǩ & $\mathrm{K}$ & $\mathrm{k}$ & $\mathrm{k}$ & $\delta \mathrm{k}$ & $(1-\delta) \mathrm{k}$ & $\mathrm{k}$ & $\mathrm{K}$ & $\dot{\kappa}$ & $c ̧$ & $\mathrm{v}$ & Ciclo de negocios \\
\hline 1900 & 107 & 0.44 & 0.56 & 48 & 50 & - & - & - & - & 0.07 & - & - & - & 0.80 & Prosperity; brief recession \\
\hline 1901 & 114 & 0.38 & 0.62 & 43 & 60 & -0.10 & 0.21 & -0.04 & 0.12 & 0.07 & 64 & - & 178 & 0.60 & Prosperity \\
\hline 1902 & 122 & 0.50 & 0.50 & 61 & 62 & 0.43 & 0.03 & 0.16 & 0.02 & 0.18 & 62 & -0.04 & 179 & 1.00 & Prosperity \\
\hline 1903 & 131 & 0.40 & 0.60 & 52 & 58 & -0.15 & -0.06 & -0.07 & -0.03 & -0.11 & 69 & 0.12 & 212 & 0.66 & Prosperity; recession \\
\hline 1904 & 138 & 0.51 & 0.49 & 70 & 70 & 0.35 & 0.20 & 0.14 & 0.12 & 0.26 & 80 & 0.16 & 196 & 1.03 & Mild depression; revival \\
\hline 1905 & 149 & 0.44 & 0.56 & 65 & 83 & -0.07 & 0.18 & -0.03 & 0.09 & 0.06 & 79 & -0.01 & 210 & 0.78 & Prosperity \\
\hline 1906 & 163 & 0.44 & 0.56 & 72 & 75 & 0.10 & -0.09 & 0.04 & -0.05 & -0.01 & 80 & 0.02 & 241 & 0.78 & Prosperity \\
\hline 1907 & 176 & 0.38 & 0.62 & 67 & 73 & -0.07 & -0.03 & -0.03 & -0.02 & -0.05 & 101 & 0.25 & 278 & 0.61 & Prosperity; panic; recession; depression \\
\hline 1908 & 185 & 0.62 & 0.38 & 114 & 93 & 0.70 & 0.27 & 0.27 & 0.17 & 0.43 & 112 & 0.11 & 217 & 1.60 & Depression \\
\hline 1909 & 198 & 0.72 & 0.28 & 142 & 72 & 0.25 & -0.22 & 0.15 & -0.08 & 0.07 & 105 & -0.06 & 249 & 2.55 & Revival; Mild prosperity \\
\hline 1910 & 208 & 0.21 & 0.79 & 43 & 126 & -0.70 & 0.74 & -0.50 & 0.21 & -0.29 & 136 & 0.29 & 314 & 0.26 & Recession \\
\hline 1911 & 216 & 0.20 & 0.80 & 42 & 61 & 0.00 & -0.51 & 0.00 & -0.41 & -0.41 & 90 & -0.34 & 380 & 0.24 & Mild depression \\
\hline 1912 & 226 & 0.40 & 0.60 & 91 & 137 & 1.14 & 1.24 & 0.22 & 0.99 & 1.22 & 165 & 0.82 & 305 & 0.67 & Revival; prosperity \\
\hline 1913 & 236 & 0.24 & 0.76 & 56 & 93 & -0.38 & -0.32 & -0.15 & -0.19 & -0.35 & 99 & -0.40 & 378 & 0.31 & Prosperity; recession \\
\hline 1914 & 244 & 0.49 & 0.51 & 120 & 122 & 1.13 & 0.32 & 0.27 & 0.24 & 0.51 & 151 & 0.53 & 331 & 0.96 & Depression \\
\hline 1915 & 266 & 0.25 & 0.75 & 66 & 124 & -0.45 & 0.01 & -0.22 & 0.01 & -0.22 & 144 & -0.05 & 407 & 0.33 & Revival; prosperity \\
\hline 1916 & 298 & 0.51 & 0.49 & 151 & 168 & 1.31 & 0.36 & 0.32 & 0.27 & 0.59 & 174 & 0.21 & 371 & 1.03 & Prosperity \\
\hline 1917 & 335 & 0.41 & 0.59 & 139 & 160 & -0.08 & -0.05 & -0.04 & -0.02 & -0.07 & 167 & -0.04 & 483 & 0.71 & Prosperity; war activity \\
\hline 1918 & 366 & 0.23 & 0.77 & 83 & 126 & -0.40 & -0.21 & -0.17 & -0.13 & -0.29 & 206 & 0.24 & 610 & 0.29 & War activity; recession \\
\hline 1919 & 387 & 0.34 & 0.66 & 130 & 212 & 0.57 & 0.68 & 0.13 & 0.53 & 0.66 & 261 & 0.27 & 550 & 0.51 & Revival; prosperity \\
\hline 1920 & 407 & 0.27 & 0.73 & 110 & 219 & -0.16 & 0.03 & -0.05 & 0.02 & -0.03 & 195 & -0.25 & 592 & 0.37 & Prosperity; recession; depression \\
\hline 1921 & 417 & 0.51 & 0.49 & 211 & 269 & 0.92 & 0.23 & 0.25 & 0.17 & 0.41 & 198 & 0.01 & 431 & 1.02 & Depression \\
\hline 1922 & 431 & 0.37 & 0.63 & 160 & 329 & -0.24 & 0.22 & -0.12 & 0.11 & -0.01 & 162 & -0.18 & 472 & 0.59 & Revival; prosperity \\
\hline
\end{tabular}

Nota. Con base en Tabla 1.

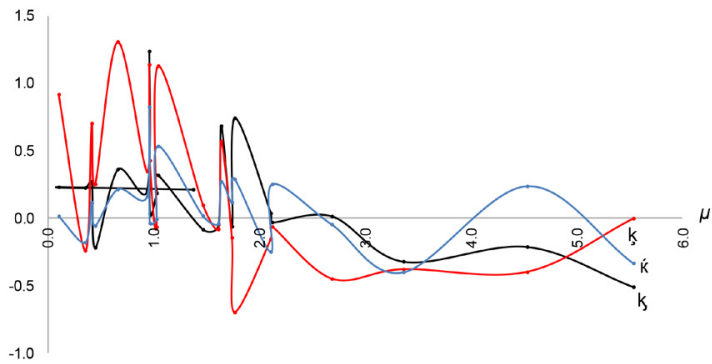

Figura 8. Tasas de variación estimada de la depreciación efectiva $\mathrm{k}$, del capital fijo remanente $\mathrm{k}$ y de la acumulación ḱ en la manufactura de los Estados Unidos (1899-1922) según $\mu$. Con base en Tabla 5. 